\title{
Idade técnica do primeiro desbaste de povoamentos de teca em diferentes espaçamentos
}

\author{
Technical age for the first thinning of teak stands in different spacings \\ Reginaldo Antonio Medeiros', Haroldo Nogueira de Paiva², Helio Garcia Leite ${ }^{3}$, \\ Thiago Taglialegna Salles ${ }^{4}$, Carlos Alberto Araújo Júnior ${ }^{5}$ e Flávio Siqueira Dávila ${ }^{6}$
}

\begin{abstract}
Resumo
Objetivou-se neste trabalho identificar a idade técnica de desbaste em povoamentos de Tectona grandis L.f. considerando diferentes espaçamentos. Os dados utilizados são oriundos de uma rede de parcelas experimentais, com áreas iguais a 1505 e $1548 \mathrm{~m}^{2}$, instaladas em dois locais no município de Água Boa, Mato Grosso, e compreendendo 12 espaçamentos. Foram consideradas nove repetições por espaçamento em delineamento inteiramente casualizado. As medições de altura total e diâmetro a 1,3 m de altura foram realizadas nas idades de 26, 42, 50 e 78 meses. Para a modelagem da distribuição diamétrica, utilizou-se a função Weibull e um conjunto de equações para estimar os parâmetros da função em uma idade futura. A idade técnica de desbaste foi definida a partir da aplicação do Método dos Ingressos Percentuais, com utilização do modelo expolinear. A estagnação do crescimento ocorreu mais cedo nos espaçamentos mais adensados, apresentando menores idades técnicas de desbaste, as quais variaram entre 6 e 8 anos. O método de ingressos percentuais e modelos de distribuição diamétrica são eficientes para definição da idade técnica de desbaste em povoamentos de teca com diferentes espaçamentos.
\end{abstract}

Palavras-chave: Tectona grandis, Método dos Ingressos Percentuais, modelo de distribuição de diâmetros, espaçamento inicial.

\begin{abstract}
The objective of this study was to identify the technical age for the first thinning in Tectona grandis L.f. stands considering different spacing. The data are from an experimental net of sampling plots, with areas equal to 1505 and $1548 \mathrm{~m}^{2}$, installed in two sites at Água Boa municipality, Mato Grosso state, considering twelve different spacing. Nine replications were considered per spacing, in a completely randomized design. Measurements of total height of the trees and diameter at $1.3 \mathrm{~m}$ in height were taken at the ages of 26, 42, 50 and 78 months. To model the diameter distribution, the Weibull function and a set of equations to estimate the values of its parameters in a future age were used. The technical age of thinning was defined based on the application of the Percentage Ingrowth Method, using the expo-linear model. Stagnation of growth occurred earlier in the denser spacing, with a lower technical age of thinning, which ranged between 6 and 8 years. The percentage ingrowth method and the diameter distribution models are efficient to define the technical age of thinning in teak stands with different spacing.
\end{abstract}

Keywords: Tectona grandis, Percent Entries Method, diameter distribution model, initial spacing.

1Professor Doutor do Curso de Engenharia Florestal. IFMT - Instituto Federal de Mato Grosso / Campus Cáceres - Prof. Olegário Baldo. Av. dos Ramires s/n - Caixa Postal: 244 - 78.200-000 - Cáceres, MT, Brasil. E-mail: reginaldo.medeiros@cas.ifmt.edu.br.

2Professor Titular do Departamento de Engenharia Florestal. UFV - Universidade Federal de Viçosa / Centro de Ciências Agrárias. Av. P. H. Hols, s/n - 36.570-000 - Viçosa, MG, Brasil. E-mail: hnpaiva@ufv.br.

3Professor Adjunto do Departamento de Engenharia Florestal. UFV - Universidade Federal de Viçosa / Centro de Ciências Agrárias. Av. P. H. Hols, s/n - 36.570-000 - Viçosa, MG, Brasil. E-mail: hgleite@gmail.com.

${ }^{4}$ Professor do Doutor do Instituto de Ciências Exatas. UNIFAL - Universidade Federal de Alfenas. Rua Gabriel Monteiro da Silva, 714 - 37.130-001 - Alfenas, MG - Brasil. E-mail: thiagotsalles@gmail.com.

5Professor Adjunto do Curso de Engenharia Florestal. UFMG - Universidade Federal de Minas Gerais / Instituto de Ciências Agrárias. Avenida Universitária, 1000 - 39.404-547 - Montes Claros, MG, Brasil. E-mail: araujocaj@gmail.com.

6Engenheiro Florestal. Companhia do Vale do Araguaia. Rua 5, 490 - 78.635-000 - Água Boa, MT, Brasil. E-mail: fdavila@valedoaraguaia.com.br. 
Medeiros et al. - Idade técnica do primeiro desbaste de povoamentos de teca em diferentes espaçamentos

\section{INTRODUÇÃO}

Na implantação de povoamentos florestais a definição do espaçamento inicial de plantio é uma das etapas mais importantes. Sua escolha, que é função do genótipo, características do local e objetivos do plantio, definirá o espaço de crescimento das árvores e influenciará nas taxas de crescimento e produção, qualidade da madeira produzida, regime de desbaste e desrama, forma do tronco e copa, sobrevivência, idade de corte, custos e rendimentos operacionais nas atividades de implantação, manutenção e colheita (BALLONI; SIMÕES, 1980; PAIVA et al., 2011; PASSOS et al., 2006)

Espécies que apresentam maior rotação, como a teca (Tectona grandis L.f.), necessitam de intervenções silviculturais periódicas, como o desbaste, ao longo do tempo (CAMPOS; LEITE, 2013). A ausência desta prática silvicultural pode afetar negativamente o crescimento e a produção dos povoamentos de teca, independente do espaçamento inicial de plantio (SILVA et al., 2016). Nesse contexto, a definição de um regime de desbaste, evidenciando o tipo, peso, técnica e época é imprescindível no plano de manejo florestal (PAIVA; LEITE, 2015; PAIVA et al., 2011).

Na definição dos regimes de desbaste é necessário utilizar modelos de crescimento e produção que incluem efeitos da densidade do povoamento e da capacidade produtiva em diferentes níveis, como, por exemplo, modelos de distribuição de diâmetro (MDD), os quais estimam a frequência e o número de árvores por área e classe de diâmetro, em idades atuais e futuras, possibilitando um monitoramento mais detalhado da estrutura diamétrica de povoamentos florestais, além de permitir análises econômicas desses povoamentos por meio da conversão de árvores em multiprodutos, que são bases para o manejo florestal (CAMPOS; LEITE, 2013; CAMPOS; TURNBULL, 1981).

Diversas pesquisas têm sido realizadas com MDD relacionados a desbaste com a finalidade de analisar tendências de crescimento e produção antes e após a sua aplicação, identificar a idade técnica e propor regimes de desbastes (NOGUEIRA et al., 2006, 2005; LEITE et al., 2006a, 2005). Na maioria das vezes a modelagem é feita empregando-se a função Weibull (WINGO, 1988), devido a flexibilidade e características desejáveis para a modelagem (ARAÚJO JÚNIOR et al., 2012; BAILEY; DELL, 1972; CAMPOS; LEITE, 2013; CAMPOS; TURNBULL, 1981; LEITE et al., 2008, 1990).

Apesar disso, o MDD não identifica a idade exata em que a estagnação do crescimento acontece, a qual por sua vez, tem sido utilizada como referência para decidir quando desbastar (LEITE et al., 2008). Muitas vezes sua determinação é feita de modo empírico, baseado em observações de campo, experiências e conhecimentos do silvicultor ou ainda conforme a demanda do mercado consumidor, objetivos do proprietário e fatores econômicos (CAMPOS; LEITE, 2013), o que pode reduzir a produtividade ao final da rotação (TONINI et al., 2010).

Garcia (1999) utilizou um modelo exponencial justaposto a um modelo linear simples, conforme Goudriaan (1994), e propôs o Método dos Ingressos Percentuais (MIP) para determinar a idade exata em que os ingressos de indivíduos em novas classes não sejam significativos, caracterizando, assim, a idade técnica de desbaste. A eficiência do método foi comprovada por Nogueira et al. (2001).

Apesar de diversas publicações envolvendo espaçamentos de plantio (LIMA et al., 2009; MACEDO et al., 2005; PASSOS et al., 2006; RONDON, 2006; SILVA et al., 2016) e desbastes (CALDEIRA; OLIVEIRA 2008; LEITE et al., 2006a; NOGUEIRA et al., 2006, 2001), pesquisas relacionadas à definição da idade técnica de aplicação do primeiro desbaste em povoamentos, com diversos espaçamentos são incipientes ou escassas. Por isso, foi conduzido este estudo, com objetivo de identificar a idade técnica de desbaste em povoamentos de teca, implantados em diferentes espaçamentos, no município de Água Boa-MT, utilizando MDD e MIP.

\section{MATERIAL E MÉTODOS}

Os dados utilizados são provenientes de dois experimentos envolvendo espaçamento em plantios de teca no município de Água Boa - MT. Os experimentos foram instalados no ano de 2009, em diferentes locais denominados talhões 30 (coordenadas geográficas médias $13^{\circ} 59^{\prime} 54^{\prime \prime} \mathrm{S}$ e $52^{\circ} 24^{\prime} 51^{\prime \prime} \mathrm{W}$ e altitude de $368 \mathrm{~m}$ ) e talhão 31 (coordenadas $14^{\circ} 00^{\prime} 33^{\prime \prime} \mathrm{S}$ e $52^{\circ} 24^{\prime} 34^{\prime \prime} \mathrm{W}$ e altitude de $378 \mathrm{~m}$ ).

O clima da região é do tipo Aw - Clima tropical, com verão chuvoso (outubro a abril) e inverno seco (maio a setembro), com pluviosidade variando de 1.800 a $2.200 \mathrm{~mm}$ anuais. O solo foi classificado pela empresa detentora da área onde se localiza os experimentos como CXd2fd: Cambissolo 
háplico Tb distrófico (alumínico), endopetroplíntico, textura média ou argilosa, mosqueado, A moderado ou fraco, relevo plano a suavemente ondulado, situado em área de captação de água (baixada) e posicionado em declive superior a 3\% (talhão 30) e LVd4d: Latossolo Vermelho distrófico típico, textura argilosa, A moderado, relevo plano a suavemente ondulado e solo posicionado em declive superior a 3\% (talhão 31).

O preparo do solo foi por meio de ripagem e subsolagem na profundidade de 90 a $110 \mathrm{~cm}$ de profundidade (talhão 30) e subsolagem na profundidade de 45 a $50 \mathrm{~cm}$ (talhão 31). Foram utilizadas mudas seminais de teca, produzidas em tubetes de $115 \mathrm{~cm}^{3}$ de capacidade. O plantio foi manual e o replantio 40 dias após o plantio. Nesta ocasião, foram aplicados $170 \mathrm{~g}$ por planta de fertilizante NPK 6:30:6.

O controle de plantas daninhas deu-se por capina manual (coroamento), capina química (aplicação de herbicida glifosato nas entrelinhas e nas linhas nos espaçamentos mais amplos), roçada semimecanizada (nas linhas de plantio) e mecanizada (nas entrelinhas). Um ano após o plantio foi realizada a desbrota.

Em cada experimento foram avaliados 12 espaçamentos, dispostos em delineamento experimental inteiramente casualizado, com nove repetições. As unidades experimentais foram compostas de parcelas de 1505 e $1548 \mathrm{~m}^{2}$ de área (Tabela 1), totalizando 18,48 ha por talhão.

Tabela 1. Espaçamento, densidade de plantas e tamanho da parcela de unidades experimentais em Água Boa-MT. Table 1. Spacing, plant density and plot size of experimental units in Água Boa- MT.

\begin{tabular}{lccccc}
\hline Tratamento & $\begin{array}{c}\text { Área por } \\
\text { planta }\left(\mathbf{m}^{2}\right)\end{array}$ & $\begin{array}{c}\text { Espaçamento } \\
(\mathbf{m})\end{array}$ & $\begin{array}{c}\text { Plantas } \\
\text { por ha }\end{array}$ & $\begin{array}{c}\text { Tamanho da } \\
\text { parcela }(\mathbf{m})\end{array}$ & $\begin{array}{c}\text { Área da } \\
\text { parcela }\left(\mathbf{m}^{2}\right)\end{array}$ \\
\hline 1 & 7,5 & $5,0 \times 1,5$ & 1334 & $43 \times 35$ & 1505 \\
2 & 7,7 & $3,5 \times 2,2$ & 1298 & $43 \times 35$ & 1505 \\
3 & 8,4 & $3,5 \times 2,4$ & 1190 & $43 \times 35$ & 1505 \\
4 & 9,1 & $3,5 \times 2,6$ & 1098 & $43 \times 35$ & 1505 \\
5 & 9,8 & $3,5 \times 2,8$ & 1020 & $43 \times 35$ & 1505 \\
6 & 12,0 & $4,0 \times 3,0$ & 834 & $43 \times 36$ & 1548 \\
7 & 15,0 & $5,0 \times 3,0$ & 667 & $43 \times 35$ & 1505 \\
8 & 16,0 & $4,0 \times 4,0$ & 625 & $43 \times 36$ & 1548 \\
9 & 18,0 & $6,0 \times 3,0$ & 556 & $43 \times 36$ & 1548 \\
10 & 20,0 & $5,0 \times 4,0$ & 500 & $43 \times 35$ & 1505 \\
11 & 24,0 & $6,0 \times 4,0$ & 416 & $43 \times 36$ & 1548 \\
\hline
\end{tabular}

As parcelas experimentais foram medidas aos 26, 42, 50 e 78 meses após o plantio. Aos 26 e 42 meses de idade mediu-se a altura total $(\mathrm{Ht})$ das dez primeiras árvores das parcelas e nas idades de 50 e 78 meses foram medidas as alturas das cinco primeiras árvores. A altura das demais árvores foi estimada por relação hipsométrica. O diâmetro (dap) de todas as árvores foi medido a 1,3 $\mathrm{m}$ de altura.

$\mathrm{Na}$ idade de 64 meses não houve medições a campo, sendo que os dados de diâmetro e altura total das árvores para cada tratamento foram estimados empregando equações obtidas por ajuste do modelo de predição do crescimento de Pienaar e Shiver (1981), conforme Campos e Leite (2013) (Equação 1):

$$
\gamma_{2}=\gamma_{1} \mathrm{e}^{\left(-\alpha\left(I_{2}^{\beta}-I_{1}^{\beta}\right)\right)}+\varepsilon
$$

em que: $y$ é o diâmetro ou altura total nas idades atual (1) e futura (2), $\alpha$ e $\beta$ os parâmetros do modelo e $\varepsilon$ o erro aleatório.

O volume com casca foi estimado pela empresa detentora da área onde localiza-se o experimento, utilizando equações volumétricas ajustadas pelo modelo de Schumacher e Hall (1933).

Para construção do MDD foi utilizada a função densidade de probabilidade ( $f d p)$ Weibull 2-P (Equação 2). A amplitude da classe de diâmetro foi de 1,0 cm e o ajuste da fdp para cada parcela e medição foi feito utilizando o software FitFD (http://neuroforest.ucoz.com).

$$
f(x)=\left(\frac{\gamma}{\beta}\right)\left(\frac{x}{\beta}\right)^{(\gamma-1)} e^{-\left(\frac{x}{\beta}\right)^{\gamma}}
$$

em que: $f(x)$ é a função densidade de probabilidade, $x$ é o centro da classe de diâmetro, $\beta$ o parâmetro de escala e $\gamma$ o parâmetro de forma da função, sendo: $x \geq 0, \beta>0$ e $\gamma>0$. 
Para distribuição de diâmetros nas classes diamétricas, os dados foram selecionados por espaçamento e talhão. Foram ajustados os modelos 3, 4, 5 e 6, sendo que o modelo 4 foi utilizado naqueles casos em que o modelo 3 não apresentava um bom ajuste.

Os ajustes foram feitos pelo método de mínimos quadrados, pelo procedimento Quasi-Newton com o software Statistica (STATSOFT, 2015).

$$
\begin{aligned}
& \gamma_{2}=\gamma_{1}\left(I_{1} / I_{2}\right)+\propto_{1}\left(1-I_{1} / I_{2}\right) q_{2}+\varepsilon \\
& \gamma_{2}=\gamma_{1}\left(I_{1} / I_{2}\right)+\propto_{2}\left(1-I_{1} / I_{2}\right)+\varepsilon \\
& \beta_{2}=\beta_{1}\left(I_{1} / I_{2}\right)+\propto_{3}\left(1-I_{1} / I_{2}\right) q_{2}+\varepsilon \\
& q_{2}=\propto_{4}+\propto_{5} q_{1}+\varepsilon
\end{aligned}
$$

em que: $\beta$ e $\gamma$ são os parâmetros da função Weibull nas idades atual $\left(\beta_{1}\right.$ e $\left.\gamma_{1}\right)$ e futura $\left(\beta_{2}\right.$ e $\left.\gamma_{2}\right)$, q é o diâmetro quadrático médio nas idades atual $\left(q_{1}\right)$ e futura $\left(q_{2}\right)$, I é a idade atual $\left(I_{1}\right)$ e futura $\left(I_{2}\right), x$ é o centro da classe de diâmetro, $\alpha_{1, \ldots,} \alpha_{5}$ são os parâmetros do modelo e $\varepsilon$ é o erro aleatório.

Nas idades atuais considerou-se o número de árvores observado (Nobs) nestas idades em cada parcela e talhão. Nas idades futuras de 84 e 96 meses não foi possível ajustar equação para estimativa do número de indivíduos nessas idades, por isso considerou-se o número de indivíduos observados na medição realizada aos 78 meses de idade.

Para recuperar o número de indivíduos por classe de diâmetro por espaçamento e talhão foi utilizada a Equação 7, que é uma transformação da função Weibull.

$$
\text { Nrec }_{x}=\left(e^{-\left(\frac{x_{i}}{\beta}\right)^{\gamma}}-e^{-\left(\frac{x_{s}}{\beta}\right)^{\gamma}}\right) N o b s_{j}
$$

em que: Nrec $_{x}$ é o número de indivíduos recuperado por classe de diâmetro na idade considerada, $x, x_{i}$ e $x_{s}$ é o centro, limite inferior e superior da classe de diâmetro, Nobs é o número de árvores observado na idade $j$ considerada.

A validação do MDD foi por comparações entre as distribuições observadas e estimadas aos 78 meses de idade, utilizando o teste $\mathrm{L} \& \mathrm{O}$, que é um procedimento estatístico proposto por Leite e Oliveira (2002).

O ingresso de árvores em novas classes de diâmetro foi calculado conforme Garcia (1999) e Nogueira et al. (2001), a partir do diâmetro diferenciador (DD), momento em que a diferença entre duas $f d p$, em ocasiões distintas, se iguala, ou seja, o ingresso de indivíduos nas sucessivas classes de diâmetro torna-se não significativo. Este momento é caracterizado pelo parâmetro $\theta_{3}$ do modelo expolinear.

Para identificar a idade técnica de desbaste (ITD), ou seja, o DD, ajustou-se o modelo expolinear (GOUDRIAAN, 1994) (Equação 8). Para seu ajuste utilizou-se os procedimentos iterativos Quasi-Newton e Simplex, com o software Statistica (STATSOFT, 2015).

$$
I P M=\left(\frac{\theta_{1}}{\theta_{3}}\right) \operatorname{Ln}\left(1+\exp ^{\left(\theta_{2}\left(l-\theta_{3}\right)\right)}\right)+\varepsilon, \quad \varepsilon \sim \operatorname{NID}\left(0, \sigma^{2}\right)
$$

em que: IPM é o ingresso percentual médio mensal de árvores, I é idade do povoamento em meses, $\theta_{1}$ é o parâmetro que indica o crescimento máximo absoluto do ingresso percentual de árvores na fase linear da curva, $\theta_{2}$ é o parâmetro que indica o crescimento máximo relativo do ingresso percentual de árvores na fase exponencial da curva, $\theta_{3}$ é o parâmetro que corresponde à idade técnica de desbaste e $\varepsilon$ é o erro aleatório.

\section{RESULTADOS E DISCUSSÃO}

O sistema de equações que compõe o MDD utilizado nesse trabalho apresentou consistência biológica ao descrever o comportamento da distribuição de diâmetros nas classes diamétricas em idades sucessivas em função dos espaçamentos. Nos espaçamentos mais adensados, o ingresso nas sucessivas classes de diâmetro ao longo do tempo foi menor se comparado aos menos adensados, assim como a estagnação do crescimento aconteceu mais precocemente. A consistência biológica de 
outros MDD também já foi comprovada por Araújo Júnior et al. (2013), Binoti et al. (2010), Leite et al. (2005, 2006a), Nogueira et al. (2005, 2006).

De acordo com o procedimento estatístico proposto por Leite e Oliveira (2002), houve diferenças entre a distribuição diamétrica observada e estimada aos 78 meses $(p<0,05)$ nos espaçamentos com 3,5 x 2,6; 3,5 x 2,8 e 4 × 3 m (talhão 30) e 3,5 x 2,8; 6 × 3 e 5 x 4 m (talhão 31). Nestes espaçamentos verificou-se semelhança entre os padrões de distribuição observada, ocorrendo maior porcentagem de indivíduos nas classes centrais da distribuição de diâmetros, como por exemplo, no espaçamento 4 × 3 m (Figura 1a). Nos demais espaçamentos a distribuição diamétrica observada foi semelhante $(p>0,05)$ à distribuição estimada pelo MDD, a exemplo do espaçamento $5 \times 1,5$ (Figura 1b).
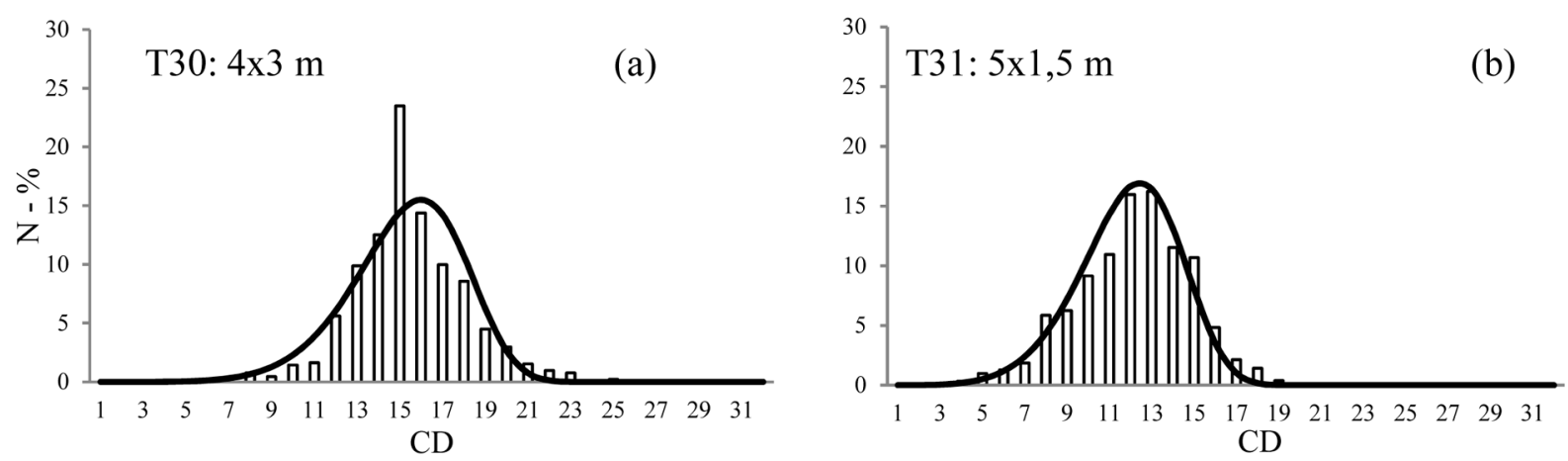

Figura 1. Porcentagem de árvores de Tectona grandis (N) observada (barras) e estimada (linha) por classe de diâmetro (CD), espaçamento e talhão $(T)$, aos 78 meses de idade.

Figure 1. Percentage of Tectona grandis (N) trees observed (bars) and estimated (line) by diameter class (CD), spacing and stand $(T)$ at 78 months of age.

O resultado significativo para o teste $L \& O$, rejeição da hipótese de que as distribuições são iguais, pode estar relacionado à utilização de uma amplitude de classe de diâmetro de $1(\mathrm{um}) \mathrm{cm}$, o que gera muitas classes (ARAÚJO JÚNIOR et al., 2010), ao uso do Teste L\&O, que é muito rigoroso (LEITE; OLIVERIA, 2002) ou as variações ocorridas nas parcelas experimentais. Por exemplo, no espaçamento 4 x 3 m (talhão 30) em sete das nove parcelas as distribuições observadas diferiram das recuperadas $(p<0,05)$, indicando a necessidade de avaliar outros MDD, de modo a buscar um modelo que proporcione distribuições diamétricas recuperadas estatisticamente iguais às observadas.

A distribuição diamétrica em todos os espaçamentos, nos dois talhões, apresentou comportamento esperado para povoamentos equiâneos, ou seja, com o passar do tempo as distribuições se achataram e deslocaram para a direita (LEITE et al., 2008) (Figura 2).

Nos espaçamentos mais adensados ( 5 x 1,5; 3,5 x 2,2;3,5 x 2,4;3,5 x 2,6;3,5 x 2,8 e 4 × $3 \mathrm{~m}$ ) verificou-se uma redução da área entre curvas de distribuição nas idades mais avançadas, devido à redução do espaço de crescimento e início da competição entre plantas, levando à diminuição dos ingressos das árvores nas sucessivas classes de diâmetro (NOGUEIRA et al., 2006). Além disso, nesses espaçamentos verificou-se que os valores da variável gama $(\gamma)$ da função Weibull diminuíram com a idade, tornando mais acentuada a assimetria das curvas de distribuição diamétrica. Isso sugere um aumento da competição entre as árvores, o início da estagnação do crescimento e a necessidade de intervenção silvicultural, como aplicação de desbaste (NOGUEIRA, 2003).

Nestes espaçamentos verificou-se também menor ingresso nas sucessivas classes de diâmetro. As curvas de distribuição diamétrica apresentaram forma mais leptocúrtica, além de abrangerem menor número de classes de diâmetro. Dentre estes, o primeiro ( 5 x 1,5 m) apresentou forma mais achatada em relação aos demais, provavelmente devido à baixa sobrevivência ou ao espaçamento entre plantas nas linhas e entrelinhas. Nos espaçamentos mais amplos $(5 \times 3 ; 4 \times 4 ; 6 \times 3 ; 5 \times 4 ; 6 \times$ 4 e $5 \times 5 \mathrm{~m}$ ) houve um maior ingresso nas sucessivas classes diamétricas com a idade. Além disso, as curvas se apresentam mais platicúrticas e abrangem maior número de classes.

Tomando como referência a classe de diâmetro com centro em $24 \mathrm{~cm}$ no talhão 30, observou-se nos espaçamentos mais adensados que em média $4 \%$ dos indivíduos estavam presentes nesta classe, ao passo que naqueles menos adensados o percentual médio foi de $30 \%$. Já no talhão 31, para a mesma classe de diâmetro, os percentuais foram de 1 e $17 \%$, respectivamente, espaçamentos mais e 
Medeiros et al. - Idade técnica do primeiro desbaste de povoamentos de teca em diferentes espaçamentos

menos adensados, o que demonstra, além do efeito do espaçamento no ingresso nas sucessivas classes de diâmetro (NOGUEIRA et al., 2006), o efeito do local (LEITE et al.,2006b; PAIVA et al., 2011).
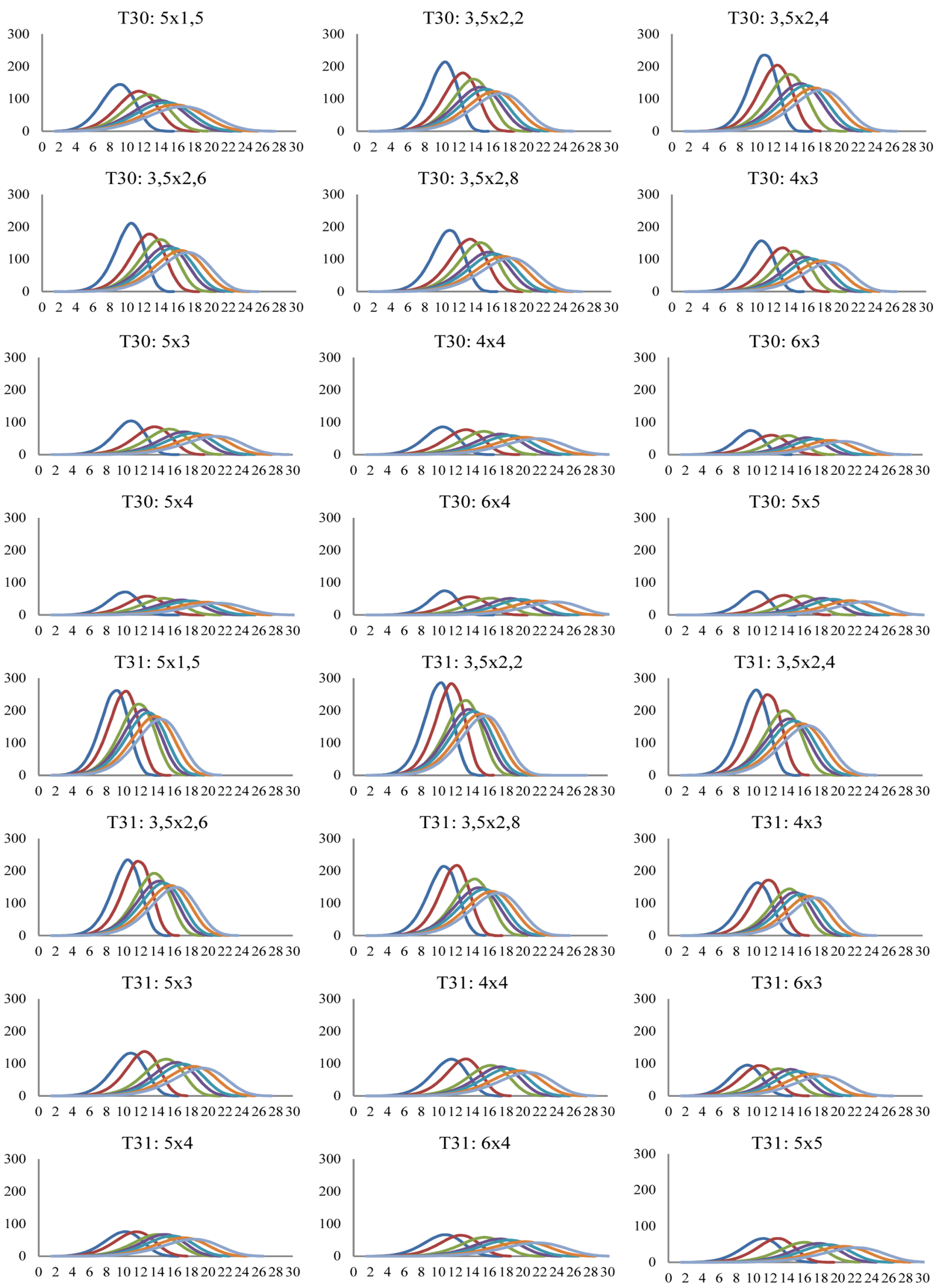

-26 meses -42 meses -50 meses _ 64 meses -78 meses -84 meses -96 meses

Figura 2. Número de árvores de Tectona grandis por hectare (eixo y), por classe de diâmetro (eixo x), em diferentes espaçamentos e talhões ( $T$ ).

Figure 2. Number of trees of Tectona grandis per hectare ( $y$ axis), by diameter class ( $x$ axis), at different spacings and stands $(\mathrm{T})$. 
Os resultados aqui obtidos corroboram com informações de Nogueira (2003), Nogueira et al. $(2005,2006 a)$, Leite et al. $(2005,2006 a)$ onde os autores comprovaram a eficiência dos MDD para análise da tendência de crescimento e produção em povoamentos submetidos a desbaste, no que se refere ao comportamento dos indivíduos antes e após a sua aplicação, definição de idade técnica e regimes de desbaste.

Observou-se que o modelo expolinear é logico e apresenta realismo biológico na determinação da idade técnica do primeiro desbaste em teca (Tabela 2). De acordo com Garcia (1999), o emprego de um modelo expolinear, relacionando o ingresso percentual mensal e a idade permite identificar o momento em que a estagnação do crescimento se inicia, sendo um indicativo da idade ótima de aplicar o desbaste. Isto tem um significado prático importante no planejamento e no plano de manejo florestal, pois diminui a subjetividade na análise de curvas de crescimento, possibilitando ao gestor florestal tomada de decisões mais acertadas, baseadas em informações mais seguras.

Nesse trabalho, preferiu-se utilizar o ingresso percentual médio mensal (IPM), conforme Nogueira (2003), ao ingresso percentual (IP) para a identificação da ITD, conforme idealizado por Garcia (1999). Além de facilitar o ajuste do modelo, esta transformação resulta em melhor descrição das fases linear e exponencial, em uma escala mensal (Tabela 2).

Neste trabalho as idades técnicas do primeiro desbaste variaram entre 6 (espaçamentos menores) e 8 anos (espaçamentos maiores), o que era esperado. A idade técnica do primeiro desbaste nos povoamentos mais adensados foi mais precoce em relação aos menos adensados, decorrente do maior número de indivíduos e, consequente, competição entre plantas por fatores de crescimento (BALLONI; SIMÕES, 1980; PAIVA et al., 2011). Verificou-se que na medida em que a área útil por planta aumenta, a idade técnica de desbaste é maior (Figura 3).

$\mathrm{O}$ método dos ingressos percentuais foi adequado para determinar a idade técnica do primeiro desbaste em povoamentos de teca implantados em diferentes espaçamentos. Em povoamentos de eucalipto e teca submetidos a desbaste, Nogueira (2003), e Nogueira et al. (2001) também comprovaram a eficiência do MIP na definição da idade técnica do desbaste. Os autores também constataram a eficiência do método na definição do segundo desbaste e na simulação de cenários envolvendo diferentes regimes de desbaste, como por exemplo, idade técnica e pesos de desbaste. Ainda de acordo com os autores, a ITD obtida no ajuste do modelo expolinear caracteriza o exato momento de mudança de fase linear para exponencial, ou seja, o momento que o ingresso de indivíduos nas sucessivas classes de diâmetro não é mais significativo, portanto, início da estagnação do crescimento e momento de aplicar o desbaste.

Maiores espaçamentos, com maior área útil por planta, implicam em maior idade técnica de desbaste. Entretanto, os resultados obtidos sugerem que a distância entre plantas nas linhas e entrelinhas, as condições de local e sobrevivência também influenciam os resultados, corroborando com Balloni e Simões (1980), Leite et al. (2006b) e Paiva et al. (2011).

Por exemplo, o espaçamento $5 \times 3 \mathrm{~m}$, com $15 \mathrm{~m}^{2}$ planta $^{-1}$, a princípio deveria apresentar maior idade técnica de desbaste que o de 4 x $3 \mathrm{~m}$, com $12 \mathrm{~m}^{2}$ planta $^{-1}$. No entanto, verificou-se que a ITD ocorrerá praticamente na mesma idade (6,5 anos). Por outro lado, o espaçamento $6 \times 3 \mathrm{~m}\left(18 \mathrm{~m}^{2}\right.$ planta $^{-1}$ de área útil), embora tenha mesma distância entre covas na linha de plantio e sobrevivência de $54 \%$, a ITD foi apenas 6 meses superior (7,1 anos), sendo que nos demais a sobrevivência foi de 72 e $78 \%$, respectivamente.

Conforme esperado, a maior distância entre linhas não implica necessariamente em uma maior idade técnica do primeiro desbaste. Entre os espaçamentos que possuem $4 \mathrm{~m}$ entre covas na linha de plantio ( 4 × 4; $5 \times 4$ e $6 \times 4 \mathrm{~m})$ a ITD foi praticamente a mesma $(7,1,6,9$ e 7,1 anos) e a sobrevivência foi de 70, 64 e $79 \%$, respectivamente.

Nos espaçamentos com 3,5 x 2,2; 3,5 x 2,6 e 3,5 x 2,8 m a ITD foi igual para todos (5,8 anos). Porém, no espaçamento 3,5 × 2,4 m, com espaçamento e área útil similar aos anteriores, a ITD foi superior (6,5 anos), sendo atribuindo esse resultado às condições do local (LEITE et al., 2006b; PAIVA et al., 2011), pois o mesmo não ocorreu no talhão 31. Ainda, a sobrevivência destes tratamentos no talhão 30 foi semelhante $(71,85,86$ e $86 \%)$.

Entre os espaçamentos com $5 \mathrm{~m}$ entre linhas ( $5 \times 1,5 ; 5 \times 4$ e $5 \times 5 \mathrm{~m})$ observou-se que a ITD aumenta a medida que aumenta o espaçamento entre plantas na linha de plantio $(6,3,6,9$ e 7,5 anos, respectivamente). 
Medeiros et al. - Idade técnica do primeiro desbaste de povoamentos de teca em diferentes espaçamentos

Tabela 2. Equações expolineares ajustadas por talhão $(T)$ e espaçamento para estimar o ingresso percentual médio mensal (IPM) e a idade técnica de desbaste em anos (ITD), em função da idade (I), em teca em diferentes espaçamentos e correspondente estatística de exatidão $(r y \hat{y})$.

Table 2. Expo-linear equations adjusted per Stand $(T)$ and spacing to estimate the monthly average percentage ingress (IPM) and the technical age of thinning in years (ITD), according to age (I), in teak under different spacings and corresponding statistic of accuracy $(r y \hat{y})$.

\begin{tabular}{|c|c|c|c|c|}
\hline Espaçamento (m) & Equação(*) & & $r y \hat{y}$ & ITD \\
\hline T30: $5 \times 1,5$ & $I P M=\left(\begin{array}{l}-0,02267 \\
-0,08201\end{array}\right)$ & $\operatorname{Ln}(1+\exp (-0,08201(I-74,59252)))$ & 89,31 & 6,3 \\
\hline T30: 3,5x2,2 & $I P M=\left(\begin{array}{l}-0,03191 \\
-0,10130\end{array}\right)$ & $\operatorname{Ln}(1+\exp (-0,10130(I-69,13647)))$ & 92,24 & 5,8 \\
\hline T30: 3,5x2,4 & $I P M=\left(\frac{-0,01880}{-0.06893}\right)$ & $\operatorname{Ln}(1+\exp (-0,06893(I-78,45199)))$ & 97,11 & 6,5 \\
\hline T30: 3,5x2,6 & $I P M=\left(\begin{array}{l}-0,03148 \\
-0,09982\end{array}\right)$ & $\operatorname{Ln}(1+\exp (-0,09982(I-69,11039)))$ & 92,62 & 5,8 \\
\hline T30: 3,5x2,8 & $I P M=\left(\frac{-0,03149}{-0.09512}\right)$ & $\operatorname{Ln}(1+\exp (-0,09512(I-69,33412)))$ & 91,52 & 5,8 \\
\hline T30: 4x3 & $I P M=\left(\frac{-0,02592}{-0.09227}\right)$ & $\operatorname{Ln}(1+\exp (-0,09227(I-78,94419)))$ & 91,91 & 6,6 \\
\hline T30: $5 \times 3$ & $I P M=\left(\frac{-0,02735}{-0,07548}\right)$ & $\operatorname{Ln}(1+\exp (-0,07548(I-77,51978)))$ & 90,38 & 6,5 \\
\hline T30: $4 \times 4$ & $\left(\frac{-0,02231}{-0,07278}\right)$ & $\operatorname{Ln}(1+\exp (-0,07278(I-85,23881)))$ & 92,09 & 7,1 \\
\hline T30: $6 \times 3$ & $I P M=\left(\begin{array}{l}-0,02227 \\
-0,05760\end{array}\right.$ & $\operatorname{Ln}(1+\exp (-0,05760(I-85,22724)))$ & 88,24 & 7,1 \\
\hline T30: $5 \times 4$ & $I P M=\left(\frac{-0,02342}{-0,06948}\right)$ & $\operatorname{Ln}(1+\exp (-0,06948(I-83,18801)))$ & 92,47 & 6,9 \\
\hline T30: $6 x 4$ & $I P M=\left(\frac{-0,02503}{-0,06091}\right)$ & $\operatorname{Ln}(1+\exp (-0,06091(I-85,18200)))$ & 90,83 & 7,1 \\
\hline T30: $5 \times 5$ & $I P M=\left(\frac{-0,02465}{-0,07271}\right)$ & $\operatorname{Ln}(1+\exp (-0,07271(I-89,84849)))$ & 88,65 & 7,5 \\
\hline T31: $5 \times 1,5$ & $I P M=\left(\begin{array}{l}-0,01963 \\
-0,07402\end{array}\right.$ & $\operatorname{Ln}(1+\exp (-0,07402(I-75,46306)))$ & 91,09 & 6,3 \\
\hline T31: 3,5x2,2 & $I P M=\left(\frac{-0,01555}{-0,03767}\right)$ & $\operatorname{Ln}(1+\exp (-0,03767(I-80,38957)))$ & 83,12 & 6,7 \\
\hline T31: 3,5x2,4 & $I P M=\left(\frac{-0,02744}{-0,08701}\right)$ & $\operatorname{Ln}(1+\exp (-0,08701(I-70,92744)))$ & 94,13 & 5,9 \\
\hline T31: 3,5x2,6 & $I P M=\left(\begin{array}{l}-0,02380 \\
-0,07580\end{array}\right.$ & $\operatorname{Ln}(1+\exp (-0,07580(I-71,71789)))$ & 91,06 & 6,0 \\
\hline T31: 3,5x2,8 & $I P M=\left(\begin{array}{l}-0,0220 \\
-0,0965\end{array}\right.$ & $\operatorname{Ln}(1+\exp (-0,09652(I-77,71515)))$ & 90,83 & 6,5 \\
\hline T31: $4 \times 3$ & $I P M=\left(\frac{-0,01988}{-0,07963}\right)$ & $\operatorname{Ln}(1+\exp (-0,07963(I-81,41645)))$ & 85,15 & 6,8 \\
\hline T31: $5 \times 3$ & $I P M=\left(\frac{-0,02073}{-0,06950}\right.$ & $\operatorname{Ln}(1+\exp (-0,06950(I-81,28831)))$ & 90,04 & 6,8 \\
\hline T31: 4x4 & $I P M=\left(\frac{-0,01725}{-0,08175}\right.$ & $\operatorname{Ln}(1+\exp (-0,08175(I-89,12598)))$ & 87,82 & 7,4 \\
\hline T31: $6 \times 3$ & $I P M=\left(\frac{-0,01408}{-0,06111}\right)$ & $\operatorname{Ln}(1+\exp (-0,06111(I-95,62883)))$ & 91,89 & 7,9 \\
\hline T31: $5 \times 4$ & $I P M=\left(\frac{-0,01154}{-0,06865}\right.$ & $\operatorname{Ln}(1+\exp (-0,06865(I-96,73794)))$ & 82,15 & 8,1 \\
\hline T31: $6 x 4$ & $I P M=\left(\begin{array}{l}-0,01434 \\
-0,06565\end{array}\right)$ & $\operatorname{Ln}(1+\exp (-0,06565(I-95,69650)))$ & 89,64 & 8,0 \\
\hline T31: $5 \times 5$ & $I P M=\left(\frac{-0,01308}{-0,08672}\right)$ & $\operatorname{Ln}(1+\exp (-0,08672(I-103,46097)))$ & 83,29 & 8,6 \\
\hline
\end{tabular}

(*)Todos os coeficientes foram significativos pelo teste $\mathrm{t}(\mathrm{p}<0,05)$.

$(*)$ All coefficients were significant in the $t$ test $(p<0.05)$. 

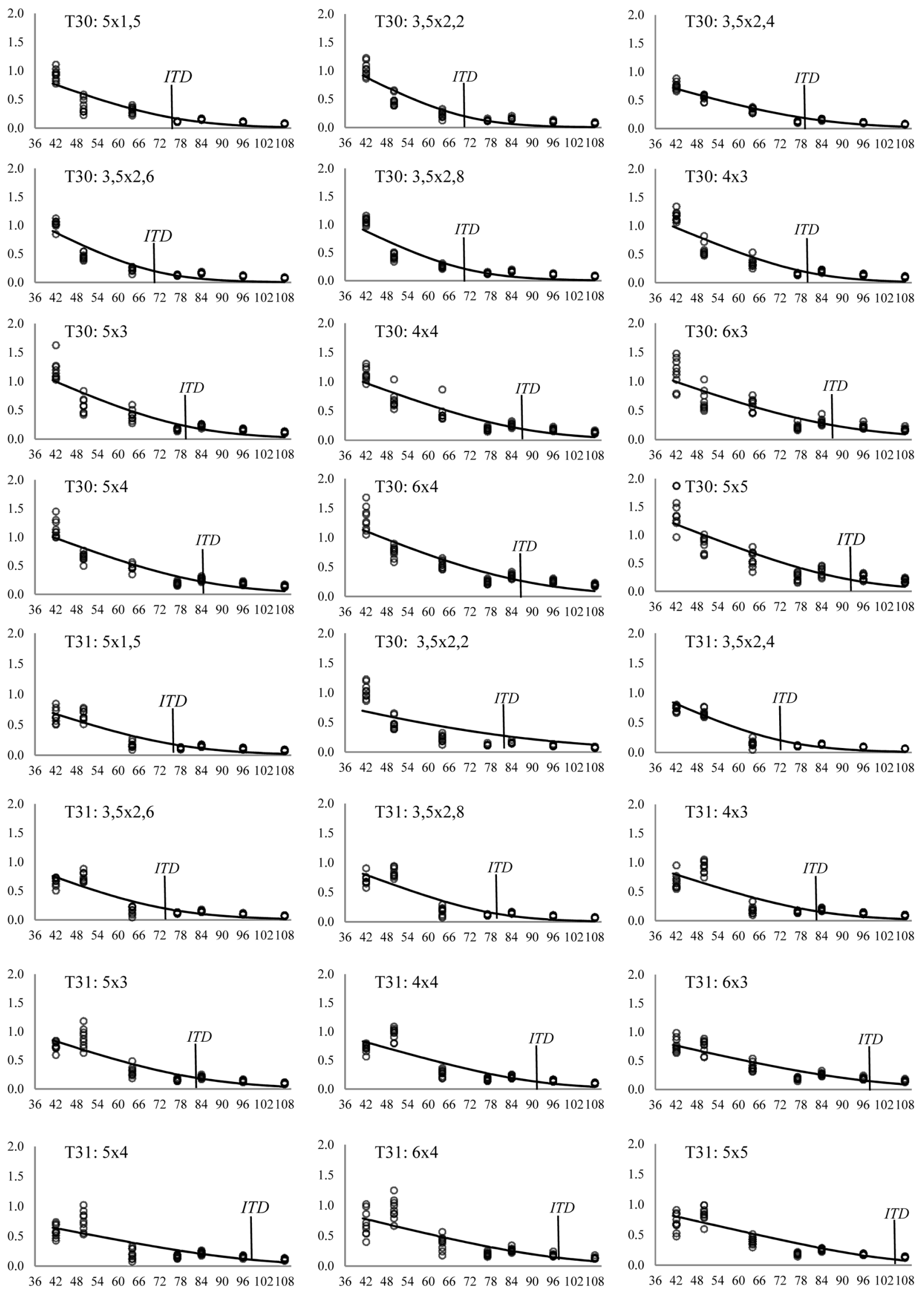

Figura 3. Idade técnica de desbaste (ITD) para Tectona grandis, indicada pela curva expolinear em diferentes espaçamentos e talhão $(T$ ), sendo: IPM=ingresso percentual mensal (eixo y) e idade em meses (eixo $x$ ).

Figure 3. Technical age of thinning (ITD) for Tectona grandis, indicated by the expo-linear curve in different spacing, stands $(T)$ where: IPM = monthly percentage ingress ( $y$-axis) and age in months ( $x$-axis). 
Além de analisar o efeito da distância entre linhas de plantio sobre a idade técnica de desbaste, os resultados obtidos sugerem que seria lógico, também, analisar a distância entre covas na linha, considerando ainda efeitos do local.

Os resultados mostraram que no talhão 30 entre os tratamentos com mesmo espaçamento, na sua maioria, a idade técnica de desbaste foi menor em relação ao talhão 31. Isso sugere que a capacidade produtiva do primeiro seja maior que a do segundo, pois, os solos onde localizam-se os talhões são diferentes. Conforme Leite et al. (2006b) e Paiva et al. (2011) em locais de maior capacidade produtiva as árvores atingem a estagnação do crescimento mais cedo, por apresentarem maior taxa de crescimento.

\section{CONCLUSÕES}

A estagnação do crescimento ocorre mais cedo nos menores espaçamentos em povoamentos de teca.

A idade técnica de desbaste aumenta à medida que aumenta a área útil por planta.

$\mathrm{O}$ ingresso das árvores nas sucessivas classes de diâmetro diminui com a idade, independente do espaçamento, sendo que nos maiores espaçamentos a diminuição é menos acentuada.

Uma maior distância entre linhas de plantio não implica necessariamente em maior idade técnica do primeiro desbaste, principalmente quando a distância entre plantas se mantem.

O modelo de distribuição de diâmetros (MDD) e o método dos ingressos percentuais (MIP) são eficientes para monitoramento do crescimento e produção e definição da idade técnica de desbaste (ITD) em povoamentos de teca.

Para o tipo de manejo atual, a idade técnica do primeiro desbaste varia de 6 a 8 anos nos espaçamentos estudados.

\section{AGRADECIMENTOS}

A FAPEMAT pela concessão de bolsa de estudo para o primeiro autor. Ao CNPq pela bolsa de produtividade em pesquisa para o segundo e terceiro autor. A empresa Companhia do Vale do Araguaia (CVA) pela cooperação, implantação, manutenção e coleta de dados experimentais.

\section{REFERÊNCIAS BIBLIOGRÁFICAS}

ARAÚJO JÚNIOR, C. A.; LEITE, H. G.; CASTRO, R. V. O.; BINOTI, D. H. B.; ALCÂNTARA, A. E. M.; SILVA BINOTI, M. L. M. Modelagem da distribuição diamétrica de povoamentos de eucalipto utilizando a função Gama. Cerne, Lavras, v.19, n.2, p. 307-314, 2013.

ARAÚJO JÚNIOR, C. A.; LEITE, H. G.; BINOTI, D. H. B.; CASTRO, R. V. O. Uso da função Weibull para descrever a área basal por classe de diâmetro de um povoamento de eucalipto. Scientia Forestalis, Piracicaba, v. 40, n. 95, p. 401-406, 2012.

ARAÚJO JÚNIOR, C. A.; NOGUEIRA, G. S.; OLIVEIRA, M. L. R.; MIRANDA, R. O. V.; CASTRO, R. V. O.; PELLI, E. Projeção da distribuição diamétrica de povoamentos de eucalipto em diferentes amplitudes de classe. Pesquisa Agropecuária Brasileira, Brasília, v. 45, n. 11, p. 1275-1281, 2010.

BAILEY, R. L.; DELL, R. Quantifying diameter distributions with the Weibull function. Forest Science, Amsterdam, v. 19, n. 2, p. 97-104, 1972.

BALLONI, E. A.; SIMÕES, J. W. O espaçamento de plantio e suas implicações silviculturais. Série Técnica IPEF, Piracicaba, v. 1, n. 3, p. 1-16, 1980.

BINOTI, D. H. B.; LEITE, H. G.; NOGUEIRA, G. S.; SILVA BINOTI, M. L. M.; GARCIA, S. L. R.; CRUZ, J. P. Uso da função Weibull de três parâmetros em um modelo de distribuição diamétrica para plantios de eucalipto submetidos a desbaste. Revista Árvore, Viçosa, v. 34, n. 1, p. 147-156, 2010. 
CALDEIRA, S. F.; OLIVEIRA, D. L. C. Desbaste seletivo em povoamentos de Tectona grandis com diferentes idades. Acta Amazônica, Manaus, v. 38, n. 2, p. 223-228, 2008.

CAMPOS, J. C. C.; LEITE, H. G. Mensuração florestal: perguntas e respostas. 4. ed. Viçosa: Editora UFV, 2013. $407 \mathrm{p}$.

CAMPOS, J. C. C.; TURNBULL, K. J. Um sistema para estimar produção por classe de diâmetro e sua aplicação na interpretação do efeito de desbaste. Revista Árvore, Viçosa, v. 5, n. 1, p. 1-16, 1981.

STATSOFT. Statistica: data analysis software system. Version 13.0. 2015. Disponível em: < http://www.statsoft. com.br/ >. Acesso em: 16 abr. 2016

GARCIA, S. L. R. Ingresso Percentil: teoria e aplicações em ciência florestal. 1999. 20 p. (não publicado).

GOUDRIAAN, J. Using the expolinear growth equation to analyse resource capture. In: MONTEITH, J. L.; SCOTT, R. K.; UNSWORTH, M. H. (Ed.). Resource capture by crops. Nottingham: Nottingham University Press, 1994. p. 99-110.

LEITE, H. G.; OLIVEIRA, F. H. Statistical procedure to test identity between analytical methods. Communications in Soil Science and Plant Analysis, New York, v. 33, n. 7/8, p. 1105-1118, 2002.

LEITE, H. G.; NOGUEIRA, G. S.; DIAS, A. N.; MONTE, M. A. Uso da mensuração florestal em pesquisa e em manejo de povoamentos submetidos a desbaste. In: SEMINÁRIO SOBRE SILVICULTURA EM FLORESTAS PLANTADAS, 3., 2008, Contagem. Anais... Contagem: SIF/DEF/UFV, 2008. p. 77-114.

LEITE, H. G.; NOGUEIRA, G. S.; CAMPOS, J. C. C.; TAKIZAWA, F. H.; RODRIGUES, F. L. Um modelo de distribuição diamétrica para povoamentos de Tectona grandis submetidos a desbaste. Revista Árvore, Viçosa, v. 30, n. 1, p. 89-98, 2006 a.

LEITE, H. G.; NOGUEIRA, G. S.; MOREIRA, A. M. Efeito do espaçamento e da idade sobre variáveis de povoamentos de Pinus taeda L. Revista Árvore, Viçosa, v. 30, n. 4, p. 603-612, $2006 \mathrm{~b}$.

LEITE, H. G.; NOGUEIRA, G. S.; CAMPOS, J. C. C.; SOUZA, A. L.; CARVALHO, A. F. Avaliação de um modelo de distribuição diamétrica ajustado para povoamentos de Eucalyptus sp. submetidos a desbaste. Revista Árvore, Viçosa, v. 29, n. 2, p. 271-280, 2005.

LEITE, H. G.; CAMPOS, J. C. C.; REGAZZI, A. J.; COUTO, L. Um modelo para estimar a produção de madeira para celulose e energia derivado da função Weibull. Revista Árvore, Viçosa, v. 14, n. 2, p. 102-118, 1990.

LIMA, I. L.; FLORSHEIM, S. M. B.; LONGUI, E. L. Influência do espaçamento em algumas propriedades físicas da madeira de Tectona grandis Linn. Cerne, Lavras, v. 15, n. 2, p. 244-250, 2009.

MACEDO, R. L. G.; GOMES, J. E.; VENTURIN, N.; SALGADO, B. G. Desenvolvimento inicial de Tectona grandis L.f. (teca) em diferentes espaçamentos no município de Paracatu, MG. Cerne, Lavras, v. 11, n. 1, p. 61-69, 2005.

NOGUEIRA, G. S. Modelagem do crescimento e da produção de povoamentos de Eucalyptus sp. e de Tectona grandis submetidos a desbaste. 2003. 145 p. Tese (Doutorado em Ciência Florestal) - Universidade Federal de Viçosa, Viçosa, 2003.

NOGUEIRA, G. S.; LEITE, H. G.; CAMPOS, J. C. C.; TAKIZAWA, F. H.; COUTO, L. Avaliação de um modelo de distribuição diamétrica ajustado para povoamentos de Tectona grandis submetidos a desbaste. Revista Árvore, Viçosa, v. 30, n. 3, p. 377-387, 2006. 
Medeiros et al. - Idade técnica do primeiro desbaste de povoamentos de teca em diferentes espaçamentos

NOGUEIRA, G. S.; LEITE, H. G.; CAMPOS, J. C. C.; CARVALHO, A. F; SOUZA, A. L. Modelo de distribuição diamétrica para povoamentos de Eucalyptus sp. submetidos a desbaste. Revista Árvore, Viçosa, v. 29, n. 4, p. 579-589, 2005.

NOGUEIRA, G. S.; LEITE, H. G.; CAMPOS, J. C. C. Determinação da idade técnica de desbaste em plantações de eucalipto utilizando o método dos ingressos percentuais. Scientia Forestalis, Piracicaba, n. 59, p. 51-59, 2001.

PAIVA, H. N.; LEITE, H. G. Desbastes e desramas em povoamentos de Eucalyptus. In: SCHUMACHER, M. V.; VIERA, M. (Ed.). Silvicultura do eucalipto no Brasil. Santa Maria: Editora UFSM, 2015. p. 83-112.

PAIVA, H. N.; JACOVINE, L. A. G.; TRINDADE, C.; RIBEIRO, G. T. Cultivo de eucalipto: implantação e manejo. 2. ed. Viçosa: Aprenda Fácil, 2011. 354 p.

PASSOS, C. A. M.; BUFULIN JUNIOR, L.; GONÇALVES, M. R. Avaliação silvicultural de Tectona grandis L.f., em Cáceres - MT, Brasil: resultados preliminares. Ciência Florestal, Santa Maria, v. 16, n. 2, p. 225-232, 2006.

PIENAAR, L. V.; SHIVER, B. D. Survival functions for site prepared slash pine plantations in the flat woods of Georgia Northern Florida. Southern Journal Forestry, Pretoria, v. 5, n. 2, p. 5 9-62, 1981.

RONDON, E. V. Estudo de biomassa de Tectona grandis L.f. sob diferentes espaçamentos no Estado de Mato Grosso. Revista Árvore, Viçosa, v. 30, n. 3, p. 337-341, 2006.

SCHUMACHER, F. X.; HALL, F. D. S. Logarithmic expression of timber-tree volume. Journal of Agricultural Research, Washington, v. 47, n. 9, p. 719-734, 1933.

SILVA, R. S.; VENDRUSCOlO, D. G. S.; ROCHA, J. R. M.; CHAVES, A. G. S.; SOUZA, H. S.; MOTTA, A. S. Desempenho silvicultural de Tectona grandis L.f. em diferentes espaçamentos em Cáceres, MT. Floresta e Ambiente, Seropédica, v. 23, n. 3, p. 397-405, 2016.

TONINI, H.; COSTA, M. C. G.; SCHWENGBER, L. A. M. Crescimento da teca (Tectona grandis) em reflorestamento na Amazônia Setentrional. Pesquisa Florestal Brasileira, Colombo, n. 59, p. 5-14, 2010.

WINGO, D. R. Methods for fitting the right-truncated weibull distribution to life-test and survival data. Biometrical Journal, v. 30, n. 5, p. 545-551, 1988.

Recebido em 02/09/2016

Aceito para publicação em 18/07/2017 